\title{
Potential Prospects of Future Medicine: Nano Medicine
}

\section{Prashansa Agrawal ${ }^{*}$}

Department of Chemistry, Case Western Reserve University, Cleveland, Ohio, 44106, USA

*Corresponding author: Prashansa Agrawal, Department of Chemistry, Case Western Reserve University, Cleveland, Ohio, 44106, USA, Tel: +1 216-368-2404; E-mail: prashansa.agrawal@case.edu

Received date: February 15, 2016; Accepted date: February 16, 2016; Published date: February 20, 2016

Copyright: () 2016 Agrawal P. This is an open-access article distributed under the terms of the Creative Commons Attribution License, which permits unrestricted use, distribution, and reproduction in any medium, provided the original author and source are credited.

\section{Editorial}

Nanomedicine is the use of nanotechnologies for the healthcare. It involves repair, construction, observation and regulation of human biological systems at the molecular level, via engineered nano-devices and nanostructures [1]. Nanomedicine is a vast industry with over 200 companies and 38 products globally. A minimum of $\$ 3.8$ billion is being invested every year in nanotechnology research and development [2]. In 2004, the sales of nanomedicines have reached up to $\$ 6.8$ billion and in April 2006, the journal 'Nature Materials' anticipated that 130 nanotech-based drugs and delivery systems are being developed universally [3]. This growing nanomedicine industry is expected to have a significant impact on the economy and medical care of the society.

At present, nanomedicines are used unanimously to improve the therapy and lives of patients suffering from a variety of disorders including ovarian and breast cancer, kidney disease, fungal infections, elevated cholesterol, menopausal symptoms, multiple sclerosis, chronic pain, asthma and emphysema [4]. Drugs generally lead to side-effects, either due to poor delivery at the actual site of disease or they may be cytotoxic, thus damaging healthy tissues and organs along with their targeted site, e.g. Cancer-specific drugs. Nanomedicines can play a significant role in drug delivery and cure of disease by ensuring sufficient amount of the drug enters the body, drug that does enter in the body should stay for appropriate time and is target-specific to the areas that require treatment.

Some of the nanomedicines that are commercially available or in human clinical trials include: Abraxane (nanoparticle albumin bound paclitaxel), approved by the U.S. Food and Drug Administration (FDA) to treat breast cancer [5], non-small-cell lung cancer [6] and pancreatic cancer [7]. Doxil was approved by the FDA for the treatment of HIV-related Kaposi's sarcoma, is now being used to also treat ovarian cancer and multiple myeloma [8]. Onivyde (liposome encapsulated irinotecan) used for the cure of metastatic pancreatic cancer, was approved by FDA in October 2015 [9]. Clinical trial is in progress since 2011 to use the C-dots (Cornell dots), which is smallest silica-based nanoparticles, as diagnostic tool to support surgeons to recognize the location of tumor cells [10]. Currently, the major areas of nanomedicine development include implantable materials and devices, biopharmaceutics and diagnostic tools. The nanomedicines can be used as:

1. Implantable materials in tissue repair and replacement (e.g., implant coatings, tissue regeneration scaffolds), structural implant materials (e.g. bone repair).

2. Implantable devices that includes assessment and treatment devices (e.g., implantable sensors, implantable medical devices), sensory aids (e.g., retina implants, cochlear implants), surgical aids (e.g., operating tools, surgical robotics- UCI Medical center has da
Vinci Surgical robotic system being currently approved for treatment of gall bladder, prostrate, colorectal, gynecological, esophageal and gastric bypass procedures).

3. Biopharmaceutics for drug delivery that include (a) drug encapsulation-nanoparticles ( 1 to $100 \mathrm{~nm}$ size) will have small pore size, improved solubility and different structural properties. This will improve the diffusion and degradation features of the encapsulated material and has been investigated in the treatment of neurological disorders like Parkinson's, Alzhemir's disease. Example of such nanoparticles include nanoscale silica and calcium phosphate. (b) Functional drug carriers-nanomaterials are involved in drug delivery as well, such as, fullerenes, dendrimers (e.g., vivagel) and nanoshells. Fullerenes are linked with antibodies and other targeting agent (drugs) in the area of HIV/AIDS, cancers and neuro-degenerative disorders. Dendrimers, because of their small size and branched structure, get the genetic material or tumor destroying therapy into a cell without triggering immune response. The nanoshell has a gold exterior layer which covers interior layer of silica and drugs. It can release tumor-specific antibodies when infrared light is administered.

4. Diagnostic tools involves genetic testing (such as, ultra-sensitive labeling and detection technologies, e.g., gold nanoparticle probes) miniature imaging devices, imaging using nanoparticle probes that can be linked to a dye which is visible on an MRI, medical nanomaterials and nanodevices (e.g., nanopores, artificial binding sites and molecular imprinting, quantum dots).

An early phase clinical trial using the platform of 'Minicell' nanoparticle for drug delivery has been tested on patients with advanced and untreatable cancer. As a platform technology, the minicell drug delivery system can be used to cure various cancers with different anti-cancer drugs along with the benefit of lower dose and less side-effects $[11,12]$. In 2014, a Phase 3 clinical trial for curing inflammation and pain after cataract surgery, and a Phase 2 trial for treating dry eye disease were initiated using nanoparticle loteprednol etabonate [13]. In 2015, the product, KPI-121 was found to yield statistically significant positive results for the post-surgery treatment [14].

Nanomedicines are striving for the distribution of a valuable set of research tools and clinically useful devices in the near future $[15,16]$. The National Nanotechnology Initiative assumes novel viable applications in the pharmaceutical industry that may include advanced drug delivery systems, new therapies, and in vivo imaging [17]. Nanomedicine research is receiving funding from the US National Institutes of Health, including the funding in 2005 of a five-year plan to set up four nanomedicine centers. Although potential future outcome of nanomedicines seem to be very promising, but their economic, social and health impact must be managed in a unified and safe manner. Their further expansion entails comprehensive technical and 
medical understanding of nanomedicines and the social as well as economic complications that obstruct their marketing. For this purpose, unproductive hurdles have to be substituted by effective filters in order to make nanomedicines more economical, effective and safer to use in long run.

\section{References}

1. Freitas RA (1999) Nanomedicine: Basic Capabilities.

2. Ratner M, Ratner D (2002) Nanotechnology: A Gentle Introduction to the Next Big Idea.

3. Nanomedicine: A matter of rhetoric? (2006) Nat Materials 5: 243.

4. Coombs RRH, Robinson DW (1996) Nanotechnology in Medicine and the Biosciences.

5. FDA (2012) Highlights of Prescribing Information, Abraxane for Injectable Suspension.

6. U.S. Food and Drug Administration (2012) Paclitaxel (Abraxane).

7. FDA Press Announcements (2013) FDA approves Abraxane for late-stage pancreatic cancer

8. Martis Elvis A, Badve Rewa R, Degwekar D Mukta (2012) Nanotechnology based devices and applications in medicine: An overview. Chronicles of Young Scientists 3: 68-73.
9. U.S. Food and Drug Administration (2015) FDA approves new treatment for advanced pancreatic cancer.

10. Gibney M (2013) Cornell nanosized 'dots' for diagnostics may now deliver drugs.

11. Suzanne Elvidge (2012) Bacterial 'minicells' deliver cancer drugs straight to the target.

12. First trial in humans of "minicells": a completely new way of delivering anti-cancer drugs (2012) European CanCer Organisation.

13. Kala Pharmaceuticals Initiates Phase 3 Clinical Trial for Treatment of PostSurgical Ocular Inflammation and Phase 2 Clinical Trial in Dry Eye Disease (2014) Kala Pharmaceuticals.

14. Kala Pharmaceuticals Announces Positive Results from Phase 3 Trial of KPI-121 in Cataract Surgery (2015) Kala Pharmaceuticals.

15. Wagner V, Dullaart A, Bock AK, Zweck A (2006) The emerging nanomedicine landscape. Nat Biotechnol 24: 1211-1217.

16. Freitas RA (2005) What is Nanomedicine? Nanomedicine 1: 2-9.

17. Coombs RRH, Robinson DW (1996) Nanotechnology in Medicine and the Biosciences. 